\title{
Adaptación del Inventario Revisado de Ansiedad Estado Competitiva-2 a POBLACIÓN DEPORTIVA ARGENTINA*
}

\section{Adaptation of the Revised Competitive State AnXiety InVentory-2 to sport ARGENTINIAN POPULATION}

\author{
Estefanía E. Caicedo Cavagnis ${ }^{* *}$, Germán L. Pereno** ${ }^{* *}$ Ricardo De la Vega Marcos $^{* * *}$
}

*Trabajo realizado en el marco de una Beca de Postgrado del Consejo Nacional de Investigaciones Científicas y

Técnicas (CONICET) otorgada a la primera autora.

"Licenciada y Doctoranda en Psicología. Becaria Doctoral del Consejo Nacional de Investigaciones Científicas y

Técnicas (CONICET) y Profesora Asistente de Metodología de la Investigación Psicológica en la Facultad de

Psicología de la Universidad Nacional de Córdoba (UNC). E-Mail: ecaicedo@psyche.unc.edu.ar

" Doctor en Psicología. Secretario Académico de la Facultad de Psicología, Profesor Titular del Curso de Nivelación y Profesor Adjunto de la Cátedra de Biología Evolutiva Humana en la Facultad de Psicología de la Universidad Nacional de Córdoba (UNC), Investigador Categoría 5 del Programa de Incentivos a la Investigación del Consejo Interuniversitario

Nacional (Ministerio de Educación de la Nación) y Director de equipos de investigación en emociones.

"Doctor en Ciencias de la Actividad Física y el Deporte, Magister en Psicología del Deporte y Neurociencia Cognitiva y

Licenciado en Psicología. Profesor Titular del Departamento de Educación Física, Deporte y Motricidad Humana en

la Facultad de Educación de la Universidad Autónoma de Madrid (UAM) y Responsable del Centro de Investigación,

Desarrollo e Innovación de Rayo Vallecano-Deyre. Madrid - España. E-Mail: ricardo.delavega@uam.es

Centro de Investigaciones de la Facultad de Psicología (CIPsi), Grupo vinculado al Centro de Investigaciones y Estudios sobre Cultura y Sociedad (CIECS).

Consejo Nacional de Investigaciones Científicas y Técnicas (CONICET)

Universidad Nacional de Córdoba (UNC)

Córdoba - Argentina.

\section{REsumEN}

La ansiedad es un proceso afectivo que recibió mucha atención en Psicología del Deporte, ya que por sus características es un ámbito fructífero para estudiar este proceso. En Argentina no se hallaron instrumentos adaptados que evalúen este constructo en ámbitos deportivos. El objetivo del estudio realizado fue adaptar el Revised Competitive State Anxiety Inventory-2 (Cox, Martens \& Russell, 2003) a una población de deportistas argentinos. Siguiendo la teoría multidimensional de la ansiedad de Martens (1974), este inventario posee 17 ítems distribuidos en tres factores: An- siedad Somática (AS), Ansiedad Cognitiva (AC) y Autoconfianza (ACF). Se realizaron tres estudios instrumentales y uno de diferencias entre grupos para analizar si existen diferencias en los niveles de ansiedad en función del sexo, deporte y modalidad deportiva (individual - grupal). El estudio de entrevistas cognitivas posibilitó la ampliación del número de ítems por factor. Así, se analizó la estructura interna de 22 ítems mediante un análisis factorial confirmatorio. El inventario replicó la estructura factorial original con 8 ítems para ACF, 7 para AS y 6 para AC. La confiabilidad presentó valores moderados a altos (de $\rho=.84$ a .96). Finalmente, se hallaron diferencias en función del 
sexo, deporte y modalidad deportiva en los niveles de AC y AS, mientras que las diferencias en ACF sólo se hallaron en función del sexo. El inventario posee adecuadas propiedades psicométricas y por lo tanto, es apropiado para la evaluación de la ansiedad estado competitiva en población deportiva argentina. Se espera que futuros estudios analicen las diferencias en los niveles de ansiedad considerando otros deportes, controlen el efecto de la edad y analicen la validez convergente del inventario.

Palabras clave: Ansiedad estado competitiva; Teoría Multidimensional de la Ansiedad; CSAI-2R; Psicología del Deporte; Deportistas argentinos.

\section{ABSTRACT}

Participating and / or competing in sports have certain characteristics that make the field of sports a productive one for the study of affective processes such as anxiety. It makes sense, then, that anxiety is a process that has received much attention in Sports Psychology since its beginnings as a discipline. Despite this, in Argentina there are currently no instruments to assess this construct in relation to sports. The aim of the present study was to adapt the Revised Competitive State Anxiety Inventory (CSAI-2R - Cox, Martens, \& Russell, 2003) for use with Argentinean athletes. The CSAI$2 R$ is composed of 17 items measuring three constructs, following Martens multidimensional anxiety theory (1974): Somatic Anxiety (SA), Cognitive Anxiety (CA), and Self-Confidence (SC). Three instrumental studies were carried out: cognitive interviews, a rating study and internal structure, and one of internal consistency. Finally, a group differences study was conducted. The cognitive interviews consisted in evaluating question comprehension by 10 athletes between the ages of 18 and 27 using a verbal probing technique. For the rating study, two experts in Sports Psychology and Psychometry judged the inventory's items in terms of their quality, as well as their congruence to the factors of interest. Lastly, for the studies of internal consistency and structure and of group differences study, the CSAI-2R was administered to 357 athletes 15 to 45 minutes prior to competing. The sample was made up of 162 females athletes and
195 males athletes between the ages of 11 and 47 $(M=21.83, S D=5.90)$ overall averaging 9.18 years practicing their respective sports and all having competed at a regional to international level at least once a year $(M=2.94, S D=2.38)$. The cognitive interviews helped clarify items, as well as add new ones (13 items) to be tested in subsequent studies. The rating study revealed complete agreement between the judges as regards congruence $(k=1.00 ; p<.05)$, although 8 items had to be eliminated due to poor quality $(<3)$. Thus, the final version the CSAI-2R replicated the original factorial structure with a total of 21 items. One item was eliminated and two had to be replaced because their factorial weight was below the established threshold of .40. Confirmatory Factor Analysis showed adequate indexes (CFI .954, TLI .948 RMSEA .062, 90\% CI .055 .070, WRMR 1.199). Internal consistency of the inventory showed moderate to high composite reliability values, (from .84 to .90). Overall, the composite reliability for CSAI-2R was .96 . Regarding sex differences, $t$ tests yielded statistical differences in the levels of CA $\left(M_{\text {men }}=11.32\right.$, $\left.M_{\text {women }}=10.12, t(347)=3.24, p<.05\right), \mathrm{SA}$ $\left(M_{\text {men }}=9.61, M_{\text {women }}=10.81, t(347)=3.81\right.$; $p<.05)$, and SC $\left(M_{\mathrm{men}}=26.01, M_{\mathrm{women}}=24.55\right.$, $t(347)=-2.87, p<.05)$. Also, sport modalities (group-individual) showed differences in SA $\left(M_{\text {individual }}=10.89, M_{\text {group }}=9.87, t(346)=2.83\right.$, $p<.05, d=.32)$ and AC $\left(M_{\text {individual }}=9.94\right.$, $\left.M_{\text {grupal }}=10.94, t(347)=-2.47, p<.05, d=-.29\right)$. Finally, ANOVA showed differences in SA, $F(3$, $345)=4.39, p<.05$ and CA, $F(3,345)=3.31$, $p<.05$ between sport type; Tukey post hoc tests showed that martial arts practitioners had higher levels of SA than soccer and hockey practitioners and lower levels of CA than field hockey practitioners. The CSAI-2R demonstrates good psychometric properties and, therefore, is an adequate instrument to assess competitive state-anxiety in Argentinian athletes. However, new instrumental studies are needed in order to check convergent validity and include new sports. Also, sociodemographic variables like age need to be taken into account in group differences studies.

Key words: Competitive state-anxiety; Multidimensional anxiety theory; CSAI-2R; Sports Psychology; Argentinian athletes. 


\section{INTRODUCCIÓN}

La práctica y / o competición deportiva poseen características que posicionan al deporte como un campo fructífero para el estudio de los procesos afectivos entre los que se encuentra la ansiedad (ANS), uno de los procesos más estudiados en Psicología del Deporte (Woodman \& Hardy, 2001 citado por Mellalieu, Hanton \& Fletcher, 2009).

$\mathrm{Su}$ estudio no estuvo exento de dificultades con respecto a la operacionalización del constructo, puesto que ha sido utilizado indistintamente con términos como estrés y / o activación (Lois Río \& Andrade Fernández, 2013). La ANS implica interpretar a la situación como una amenaza, mientras que la activación no involucra una interpretación (Hammermeister \& Burton, 2001). La ANS es una variedad de respuesta al estrés; es una respuesta emocional a una situación percibida como aversiva que activa mecanismos de evitación. Así, está caracterizada por la preocupación y aprensión ante la posibilidad de que exista un daño (físico y / o psicológico) que va acompañado por un incremento en la activación fisiológica como resultado de la evaluación de amenaza (Lois Río \& Andrade Fernández, 2013). La ansiedad es un estado que produce un sentimiento de inseguridad que actúa en dos planos: físico y / o psíquico y que, por tanto, genera síntomas como sentimientos de desasosiego, miedos difusos, inseguridad o reacciones motoras como temblores, dolores musculares, tics nerviosos, entre otros (Botelho Vinhais \& Salselas, 2013).

La ANS aparece ante demandas objetivas del entorno interpretadas como amenazantes por ejemplo en el caso de una competición deportiva, sea en niveles iniciales o en el alto rendimiento (Azevedo-Cruz, 1996) y resulta de un desequilibrio entre la demanda y la capacidad de respuesta percibida por el individuo. Esto se debe a que supone un juicio personal así como un juicio del entorno próximo del deportista (pares, entrenadores, familia, etc.) acerca de su capacidad para hacer frente a la competición y el resultado que derivará de ella (Gaetano, Gómez \& Altavilla, 2015).
La competición es una situación que trae aparejada incertidumbre sobre el resultado; así, a mayor incertidumbre e importancia concedida al resultado, mayor será la percepción de amenaza y por tanto, mayores serán los montos de ansiedad (Andrade Fernández, Lois Río \& Arce Fernández, 2007). Son numerosos los factores que inciden en la percepción de amenaza: la situación deportiva, el lugar de desarrollo de la competencia, la relación deportista-entrenador, el nivel de rendimiento del adversario, etc. (Botelho Vinhais \& Salselas, 2013; Gaetano et al., 2015; Kumar, 2016).

Para evaluar la ANS en contextos deportivos, el Inventario de Ansiedad Rasgo-Estado (State-Trait Anxiety Inventory - STAI) de Spielberger, Gorsuch \& Lushene (1970) ha sido el más utilizado en Psicología del Deporte ya que inicialmente, era la única medida de autoinforme que podía utilizarse en muestras no clínicas. Recién en los años 90 comenzaron a desarrollarse medidas específicas para evaluar fenómenos afectivos en el deporte (Ekkekakis, 2012). Martens desarrolló dos instrumentos de evaluación de la ANS desde la teoría multidimensional de la ansiedad (Martens, 1974): el Sport Competition Anxiety Test (SCAT - Martens, 1977) para evaluar ansiedad-rasgo y el Competitive State Anxiety Inventory (CSAI - Martens, Burton, Rivkin \& Simon, 1980) para evaluar ansiedad-estado; recogiendo así la dualidad que presentan los procesos ansiógenos (Ramis, Torregrosa \& Cruz, 2013).

El CSAI en sus distintas versiones es uno de los inventarios más utilizados en Psicología del Deporte; ha sido traducido y adaptado a distintos idiomas como el español, francés, griego, etcétera y se han utilizando para ello muestras amplias y diversas (atletas, futbolistas, luchadores, surfistas, etc.). Asimismo, ha sido empleado para diversos fines que pueden agruparse en tres ejes: (a) la relación de la ANS con otros constructos psicológicos o con competencias físicas como la resistencia y / o frecuencia cardíaca, (b) los efectos que ésta tiene sobre el rendimiento deportivo y (c) los efectos de distintos tipos de entrenamiento psicológico sobre la ANS como 
la relajación progresiva y / o imaginería (Arruza Gabilondo, González Rodríguez, Palacios Moreno, Arribas Galarraga \& Cecchini Estrada, 2010).

Cabe destacar que si bien el inventario surge como una medida unidimensional específica de la ansiedad-estado en contextos deportivos a partir de la modificación del STAI, estudios posteriores determinaron que la ANS es multidimensional no sólo por sus dimensiones de rasgo y estado sino también por las dimensiones cognitiva y somática (Hardy, 2001 citado por Lois Río \& Andrade Fernández, 2013; Jones, 1995). Así, se denomina ansiedad cognitiva (AC) a todas aquellas expectativas negativas y preocupaciones sobre uno mismo, la situación y sus consecuencias mientras que la ansiedad somática (AS) se refiere a la percepción de los elementos fisiológicos-afectivos de la experiencia ansiógena, es decir, la activación autonómica y sensaciones displacenteras como la aceleración de la tasa cardíaca, la dificultad para respirar, temblores en las manos, etc. (Martens, Vealey \& Burton, 1990).

Esta conceptualización dimensional se debe a que existen factores externos que intervienen en la valoración de la ANS precompetitiva y que hacen necesario que además de abordarse multidimensionalmente deba estudiarse como un proceso que resulta de una serie de antecedentes situacionales y disposicionales (Pineda Espejel, Lopez-Walle \& Tomas, 2015) que influyen de manera diferencial en el comportamiento. De este modo, estudiarlos separadamente posibilita comprender mejor los efectos de éstos sobre el rendimiento deportivo (Gould, Greenleaf \& Krane, 2002 citado por Lois Río \& Andrade Fernández, 2013).

Al respecto, tres líneas de investigación muestran la distinción conceptual entre AC y AS (Botelho Vinhais \& Salselas, 2013): La primera es la evidencia empírica que indica que son provocados por distintos tipos de antecedentes. Por ejemplo, la expectativa de éxito intervendría en los montos de AC y estar en el vestuario preparándose, en los de AS (Mellalieu et al., 2009). La segunda es la independencia conceptual de ambos compo- nentes, no sólo lo que se refiere al tiempo de duración de ambos sino también en relación con los efectos que tienen sobre el rendimiento en su magnitud y forma (lineal y / o curvilínea). La tercera es la evidencia vinculada a los distintos métodos utilizados para reducir los niveles de ANS en personas que manifiestan predominantemente uno $\mathrm{u}$ otro tipo de ANS.

Por ello, se desarrolló una segunda versión del instrumento que considerara los componentes de la respuesta de ansiedad y superara la unidimensionalidad de la primera versión, el CSAI-2. En los estudios de validez desarrollados, la Subescala de AC se subdividía en dos escalas, una que agrupaba los ítems formulados en negativo y otra los formulados en positivo. El primer grupo conformó la Subescala de AC y el último grupo la Subescala de Autoconfianza (ACF), entendida como el grado de certeza que tienen los deportistas sobre su habilidad para obtener éxito en su deporte y lograr lo que se proponen (Pinto \& Vázquez, 2013) y que, de acuerdo con la teoría multidimensional, tendría una relación positiva con el rendimiento, contrario a la AC (Lois Río \& Andrade Fernández, 2013). Sería un factor moderador del aumento o disminución de los niveles de ANS y, por tanto, su presencia en elevados niveles se asocia positivamente con altos niveles de rendimiento deportivo (Pinto \& Vázquez, 2013).

No obstante, este inventario fue revisado debido a una serie de limitaciones, por ejemplo, la falta de confirmación de la estructura factorial (Cox, Martens \& Russell, 2003) dio lugar a la versión revisada (CSAI-2R) que posee 17 ítems distribuidos en tres factores (subescalas): AS, AC y ACF y con formato de respuesta Likert de cuatro puntos (Nada Muchísimo) en función de cómo se siente la persona en el momento de la competencia. La puntuación mínima es de 9 y la máxima de 36 puntos para cada factor. El factor AC se evalúa mediante cinco ítems por lo que su puntuación global oscila entre 5 y 20 puntos. La consistencia interna de la escala es .81 . La Subescala de AS de siete ítems varía entre 7 y 28 puntos y su consistencia interna es 
igual a .81. Por último, la Subescala ACF conformada por cinco ítems oscila entre $5 \mathrm{y}$ un máximo de 20 puntos. Su grado de consistencia interna es .86. Finalmente, el AFC mostró un ajuste adecuado al modelo propuesto $\left[\chi^{2}(116)=228 ; p<.0001 ; \mathrm{CFA}=.95\right.$, $\mathrm{NNFI}=.94$ y $\mathrm{RMSEA}=.054]$. Las correlaciones entre factores fueron igual a .61 para AC y AS y -.58 para AC y ACF, y -.35 para AS y ACF (Cox et al., 2003).

Se hallaron dos adaptaciones españolas y una portuguesa de este inventario. La de Lois Río y Andrade Fernández (2013) utilizó procedimientos de análisis factorial exploratorio y confirmatorio que replicaron la estructura factorial original y obtuvieron valores de consistencia interna superiores a .79. El ítem 15 Mis manos están sudorosas presentó pesos factoriales por debajo del establecido. La adaptación de Arruza Gabilondo y colaboradores (2010) intentó generar una versión que posibilitara una aplicación web del inventario. Señalaron como limitaciones de su estudio el tamaño de la muestra, el tipo de muestreo utilizado, la dispersión en cuanto a la edad de los participantes y el desequilibrio en función del sexo.

Finalmente, Botelho Vinhais y Salselas (2013) utilizaron un procedimiento de análisis factorial exploratorio que replicó la estructura factorial original con un total de 16 ítems y mostró adecuados valores de consistencia interna que oscilaron entre .69 y .83 . Eliminaron el ítem Sinto o meu corpo rígido debido a que poseía cargas compartidas entre los tres factores del inventario.

Las normas de adaptación de pruebas indican que los procesos de adaptación dependen del contexto y la cultura objetivo del test (American Educational Research Association, 2014; Muñiz \& Hambleton, 1996), por lo que el objetivo de este trabajo fue contribuir a la medida de los procesos afectivos, específicamente del constructo ansiedad-estado competitivo en el contexto deportivo de Argentina intentando superar algunas limitaciones mencionadas por Arruza Gabilondo y colaboradores (2010), tales como el tamaño de la muestra y el equilibrio entre tamaño muestral, sexo y nivel deportivo.
Mediante tres estudios instrumentales se adaptó a población deportiva argentina el CSAI-2R. Por último, se estudiaron las diferencias entre grupos para analizar si existen diferencias en los niveles de ANS en función del sexo, deporte y modalidad deportiva (individual - grupal). Esto se debe a que el rol de variables socio-demográficas sobre la ANS debe determinarse (Parnabas, 2015) ya que se han encontrado algunas diferencias en función del sexo (Pineda Espejel et al., 2015), nivel de experiencia (Botelho Vinhais \& Salselas, 2013), deporte (Jones, 1995), etc.

Principalmente las investigaciones se han centrado en varones, lo que hace difícil determinar los niveles de ANS y rendimiento deportivo en deportistas mujeres; y las que sí lo hicieron hallaron diferencias no sólo en los antecedentes sino también en los componentes de la ANS en función del sexo (Parnabas, 2015).

Un estudio halló que los deportistas varones obtuvieron puntajes más bajos en ansiedad-estado competitiva que las mujeres y que ellas mostraron los niveles más bajos de rendimiento deportivo (Parnabas, 2015). Kumar (2016) por su parte, evaluó la ANS pre-competitiva mediante el SCAT entre mujeres y varones de 18 a 24 años que participaban en deportes individuales (atletismo, arquería y boxeo) y de equipo (cricket) y halló diferencias en las medias de ambos grupos, aunque no fueron significativas. Además, Pineda Espejel y colaboradores (2015) hallaron diferencias en ACF y dirección de la ANS en función del sexo. Finalmente, Botelho Vinhais y Salselas (2013) indicaron que tanto varones como mujeres poseen como valor medio más elevado el factor ACF y el más bajo en AC aunque no hallaron diferencias estadísticamente significativas en función del sexo de los participantes.

Una posible explicación a esta diferencia en función del sexo puede deberse a que las mujeres poseen mayor disposición para informar sentimientos displacenteros que hacen esperable que informen con más exactitud sus síntomas puesto que gozan de mayor aceptación social (Lois Río \& Andrade Fernández, 2013). 
Tradicionalmente se ha considerado a la naturaleza del deporte como una variable moderadora de la intensidad e interpretación de los síntomas de ANS (Jones, 1995; Martens et al., 1990). Gaetano y colaboradores (2015) al comparar deportes individuales y colectivos, encontraron que los atletas de los deportes individuales estaban significativamente más ansiosos que los que practican deportes de equipo. Kirkby y Liu (1999) hallaron diferencias con respecto a la ANS y la modalidad deportiva; los que compiten en deportes individuales presentan puntuaciones significativamente menores en $\mathrm{ACF}$ y más elevadas en AS que los que practican deportes de equipo. Aún más, Lois Río y Andrade Fernández (2013) encontraron que los karatecas mostraron niveles mayores de AS que los jugadores de fútbol sala, quienes obtuvieron niveles más altos de $\mathrm{ACF}$.

En otro estudio se encontró que en las modalidades colectivas e individuales el valor medio más elevado se halló en el factor ACF y el menor, en AC. Además, los niveles de AC y AS en las modalidades individuales son superiores a los de las colectivas, aunque las diferencias halladas no son significativas (Botelho Vinhais \& Salselas, 2013). Por su parte, Ramis, Torregrosa y Cruz (2013) tomando una muestra de niños hallaron que los deportistas que practican deportes individuales mostraron niveles de ANS más altos que los que realizan deportes colectivos. Además, al comparar los niveles de ANS entre deportes de contacto (lucha libre) y no contacto (gimnasia) no encontraron diferencias significativas. Sin embargo, estas diferencias aparecieron cuando se analizó el efecto de interacción entre estas variables (contacto - no contacto) y modalidad deportiva (individual - colectivo), lo cual fue interpretado a partir de que los deportes de contacto individuales implican niveles más altos de ANS que los deportes de contacto colectivos, mientras que en los deportes de no contacto lo individual - colectivo tiene un efecto mucho más difuso en los niveles de ANS.

A partir de estos antecedentes, se pusieron a prueba las siguientes hipótesis:

1.- Los varones poseen menores niveles de ANS general así como de AC y AS que las mujeres y mayores niveles de ACF que ellas.

2.- Los deportistas de artes marciales poseen niveles más bajos de $\mathrm{AC}$ y mayores niveles de ANS general y AS que los jugadores de fútbol y hockey.

3.- Los deportistas de deportes colectivos presentan mayores niveles de ACF que los de modalidades individuales y

4.- los deportistas que practican deportes individuales poseen mayores niveles de ANS general, AS y AC que los deportistas de deportes colectivos.

\section{MÉTOdo}

\section{FASE 1}

TRADUCCIÓN DEL INVENTARIO Y ESTUDIO DE ENTREVISTAS COGNITIVAS

Para el proceso de traducción participaron cuatro traductoras de sexo femenino (tres nativas argentinas y una nativa norteamericana), todas con conocimientos de los idiomas, culturas objetivo del inventario, del proceso de adaptación de un test y de la disciplina y población meta.

Se utilizó un proceso de traducción inversa y se solicitó por e-mail que tradujeran los ítems. Una vez enviadas las traducciones se compararon y unificaron en una versión tomando como criterio que se respetara el significado original del ítem más que la traducción literal a fin de mantener cierta equivalencia conceptual, semántica y funcional (Mimura \& Griffiths, 2008). Posteriormente, se envió la versión definitiva a la traductora norteamericana para que la tradujera nuevamente al inglés. Al finalizar se le envió al autor principal del inventario la traducción al inglés junto con los ítems originales para que juzgara la pertinencia y bondad de la traducción. Finalmente, se elaboró la versión definitiva para utilizarla en el estudio de entrevistas cognitivas que dio inicio al estudio de las propiedades psicométricas del inventario mediante un estudio de validez basado en el proceso de respuestas (American Educational Research Association, 2014). 
Tal como indicaron Ramis y colaboradores (2013), para evitar sesgos potenciales por cuestiones de género, los adjetivos se redactaron contemplando estas particularidades, por ejemplo el ítem 2 se tradujo como "Estoy preocupado/a de no desempeñarme en esta competición tan bien como podría hacerlo".

Las entrevistas cognitivas son un procedimiento que cada vez se utiliza con mayor frecuencia para la adaptación de un test. Revela cómo la población objeto de un estudio comprende un ítem determinado y por qué responde como lo hace. La premisa es que las palabras y lo que los participantes dicen durante el proceso de resolución de los ítems provee información valiosa acerca de qué se está pensando al momento de responder un cuestionario y esto puede ayudar a los investigadores a comprender cómo funcionarán los ítems y por qué funcionan de determinada manera (Almond et al., 2009 citado por Vaiman, 2011). En este estudio se hizo foco en el proceso de comprensión de la pregunta mediante la metodología del sondeo verbal para evaluar la intencionalidad de las preguntas, es decir, a qué cree el examinado que la pregunta está dirigida y cuál es el significado atribuido a los términos empleados en la misma.

Para estas entrevistas participaron $10 \mathrm{de}$ portistas de ambos sexos (5 mujeres y 5 varones) de 18 y 27 años $(M=21.5$ años; $D E=2.41)$, con una práctica deportiva desde 1 a 19 años $(M=9.94$ años $D E=6.52)$. Tres practican fútbol, 2 hockey, 1 wing tsu, 1 escalada deportiva, 1 handball y 2 tenis. El muestreo fue no probabilístico accidental (Bologna, 2010).

Se utilizó una planilla de datos sociodemográficos y deportivos para documentar variables tales como edad, sexo, puesto táctico, años de práctica de su deporte, cantidad de días de entrenamiento y horas de práctica diaria, etc. y el CSAI-2R.

Las entrevistas tuvieron una duración de una hora y fueron registradas mediante un grabador de voz. Se indagó acerca de la comprensión de los ítems que la investigadora consideró que podrían tener dificultades o múltiples interpretaciones como el ítem 10 Estoy seguro de que voy a desempeñarme bien o la comprensión de expresiones idiomáticas como tengo un nudo en el estómago.

Las entrevistas se transcribieron en una tabla de doble entrada en la cual las respuestas a cada pregunta específica fueron sintetizadas tomando en consideración el sexo de los participantes y posteriormente se unificaron estas respuestas para compararlas por sexo y evaluar diferencias en la comprensión en función de esta variable. Los participantes manifestaron su interés en participar voluntariamente y antes de iniciar las entrevistas firmaron una nota de consentimiento informado.

A partir de este procedimiento, se modificaron, profundizaron y mejoraron los ítems. También brindaron información pertinente para la elaboración de nuevos reactivos ya que se buscó que cada factor del inventario tuviera 7 ítems como mínimo para balancear la cantidad de ítems por factor y que la cantidad fuera representativa del factor. Así, por ejemplo, se solicitó a los participantes que brindaran tres indicadores de que el cuerpo está tenso, y con ese aporte se generó el ítem Siento que mis músculos están duros. Sobre las expresiones idiomáticas como tener un nudo en el estómago dijeron que quiere decir estar nervioso, no poder tramitar la ansiedad $y$ sentirse angustiado. Al finalizar este estudio el inventario quedó conformado por 30 ítems, 10 para cada factor, y se procedió a estudiar la validez de contenido.

\section{ValideZ dE CONTENIDO. ESTUDIO dE JUECES}

A dos jueces expertos en Psicometría y Psicología del Deporte se les entregó una planilla con un formato estandarizado de clasificación de ítems que incluía los 30 ítems y los criterios de evaluación para que los valoraran en función de su calidad semántica, gramatical y su congruencia con el constructo teórico que se pretende medir. Para ello se solicitó que juzgaran a cuál de los factores del CSAI-2R pertenecía cada ítem del inventario y su calidad en un formato Likert de cuatro puntos, siendo 1: muy inadecuada que indicaría una baja calidad y una sugerencia im- 
plícita de reelaboración del mismo y 4: muy adecuada que indicaría una alta calidad. Finalmente cada juez podía indicar las observaciones que pudiera tener con respecto a los ítems más allá de las valoraciones de calidad y congruencia.

Se utilizó el coeficiente Kappa $(k)$. Su puntaje va de 0 a 1 , donde 1 indica un acuerdo perfecto (Mc Hugh, 2012). Este coeficiente arrojó un índice de concordancia igual a 1.00 $(p<.05)$ es decir, un acuerdo perfecto. Sin embargo, debido al bajo puntaje $(<3)$ en relación a su calidad y a las observaciones de los jueces que recibieron 8 de los 13 ítems generados posteriormente a las entrevistas cognitivas, se decidió eliminarlos para los siguientes estudios. Quedó, por tanto, un inventario de 22 reactivos que fue sometido a análisis de estructura y consistencia interna.

\section{FASE 2}

ANÁLISIS DE LA ESTRUCTURA Y CONSISTENCIA INTERNA DEL CSAI-2R

\section{PARTICIPANTES}

Participaron 357 deportistas de ambos sexos, 162 mujeres $(45.4 \%)$ y 195 varones (54.6\%) (ver Tabla 1), de 11 a 47 años $(M=21.83 ; D E=5.90)$. De ellos, 14 practican tenis, 92 artes marciales, 123 fútbol y 128 hockey. Las horas de entrenamiento diario oscilan de 1 a 9 horas como máximo $(M=2.34$; $D E=.89)$, entrenan entre uno y siete días por semana $(M=3.43 D E=1.15)$.

Practican su deporte hace 9.18 años $(D E=6.43)$ y compiten al menos una vez al año $(M=2.94 ; D E=2.38)$ en los niveles universitario $(n=9)$, provincial $(n=113)$, regional $(n=72)$, nacional $(n=92)$ e internacional $(n=59)$. La estrategia de muestreo fue accidental (Bologna, 2010).

\section{INSTRUMENTOS}

1.- Planilla de datos sociodemográficos y deportivos: En ella se registraron variables ta- les como edad, sexo, puesto táctico, años de práctica de su deporte, cantidad de días de entrenamiento y horas de práctica diaria, etc.

2.- Competitive State Anxiety Inventory-2R (Cox et al., 2003): A partir de los procesos descriptos anteriormente este inventario quedó conformado por 22 ítems. Tanto el factor AC como AS poseen siete ítems que puntúan entre 7 y 28 puntos como máximo y el factor ACF tiene ocho ítems con una puntuación de 8 a 32.

\section{Procedimiento}

Se estableció contacto con la Confederación de Deportes de la Provincia de Córdoba, clubes y entrenadores para invitarlos a participar mediante una nota en la que se explicaban objetivos, detalles y requerimientos del estudio. A quienes estuvieron dispuestos a participar se les solicitó un calendario de competiciones y se coordinó una fecha para evaluar a los deportistas lo más cerca posible de la competencia (entre 45 y 20 minutos antes) de acuerdo a lo sugerido por Andrade Férnandez y colaboradores (2007).

Se administró el inventario colectivamente para los deportes de equipo y de manera personal para los individuales, en un lugar tranquilo (vestuarios o inmediaciones del lugar de competencia), con buena iluminación y con la supervisión de la investigadora. Previo a la entrega de los cuestionarios se realizaba una presentación del estudio, consignas y modo de completarlo; también se indicaba que todo aquel que quisiera podía solicitar un informe del estudio y que una vez finalizada su participación se le brindaría toda la información que desee. La administración duró 10 minutos y al finalizar la fase de adaptación se envió un informe del proceso a quienes lo solicitaron por correo electrónico. Todos los participantes manifestaron su interés en participar del estudio voluntariamente y firmaron una nota de consentimiento informado donde se explicitó la anonimidad y confidencialidad de la información, y que su participación no implicaba riesgo para su salud, entre otros. 


\section{ANÁLISIS DE DATOS}

Se utilizó el software SPSS 19 para preparar los datos y se realizó una exploración inicial de ellos. Primero se evaluó el patrón de los valores perdidos con el fin de estimar si el mismo respondía a una distribución aleatoria (Tabachnick \& Fidell, 2011). Luego, dado que la correlación inter-ítems de los factores del inventario fue superior a .30, se analizaron los siguientes datos por factor del inventario y así, se identificaron los casos atípicos. Para los univariados se calcularon las puntuaciones $z$ y se inspeccionaron los datos mediante los gráficos de cajas y bigotes, considerándose atípicos aquellos datos con puntuaciones $z> \pm 3.29(p<.001)$ y que fueran identificados como tales en los gráficos. Para los multivariados se utilizó la prueba de distancia de Malahanobis $(D)$ considerándose atípico todo caso que presentara en dicha prueba una significación menor o igual a .01, debido al largo número de evaluaciones involucradas (Meyers, Gamst \& Guarino, 2013).

A continuación se evaluó el tipo de distribución de las puntuaciones mediante los índices de asimetría y curtosis y $Q-Q$ plots ya que las pruebas de normalidad son sensibles al tamaño de la muestra y ante pequeñas variaciones en muestras grandes tienden a mostrar niveles de significación menores o iguales a .05 (Wilcox, 2003 citado por Larson Hall, 2010). Se consideraron valores excelentes, índices de asimetría y curtosis entre \pm 1.00 y como adecuados, valores inferiores a \pm 2.00 (George \& Mallery, 2011). Después se indagó mediante el estadístico de Levene la homogeneidad de la variancia. Este supuesto indica que las medias de las puntuaciones pueden diferir pero que las variancias deben ser iguales (Larson Hall, 2010).

Para evaluar la evidencia de la estructura interna se realizó un análisis factorial confirmatorio (AFC) con el programa Mplus versión 6.12 puesto que permite aplicar el método de estimación de parámetros Robust Weighted Least Squares considerado como el más pertinente para analizar datos categóricos (por ejemplo, escalas tipo Likert). Para eva- luar el ajuste del modelo se utilizó el estadístico chi-cuadrado, el índice de ajuste comparativo (CFI), el índice de Tucker-Lewis (TLI), el error cuadrático medio de aproximación (RMSEA) y la media cuadrática residual ponderada (WRMR). Los valores entre .90 y .95 o superiores para CFI y TLI son considerados como ajuste aceptable a excelente. Para RMSEA se esperan valores entre .05 y .08 , y para WRMR valores menores a 1.00 (Yu \& Muthen, 2002). Con respecto a la confiabilidad, se estimó la fiabilidad compuesta, la cual utiliza las cargas factoriales, varianza residual y $r^{2}$ de cada ítem según una estructura subyacente, presentando menor variancia de error. Un índice igual o superior a $\rho=.70$ es considerado aceptable (Nunnally, 1978).

Finalmente, con el objetivo de analizar las diferencias entre los participantes del estudio en función del deporte practicado, se realizó un análisis de la variancia (ANOVA), mientras que para analizar las diferencias en los niveles de ANS en función de la modalidad deportiva y el sexo se realizaron dos pruebas $t$ de diferencias entre muestras independientes. Se analizaron los tamaños del efecto con el estadístico $d$ de Cohen para las pruebas $t$ y mediante el coeficiente eta cuadrado parcial $\left(\eta^{2}\right)$ el ANOVA. Se consideraron valores pequeños $\left(d=|.2|, \eta^{2}=1 \%\right)$, medianos $(d=|.5|$, $\left.\eta^{2}=10 \%\right)$ y grandes $\left(d=|.8|, \eta^{2}=25 \%\right)(\mathrm{Co}-$ hen, 1988).

\section{Resultados \\ ANÁLISIS DE ÍTEMS}

El porcentaje de valores perdidos no superó el 5\% establecido por la literatura (Graham, 2009) y se distribuyó en diferentes variables, por lo tanto se decidió imputar estos datos por la moda de las respuestas completas de un participante en una misma subescala ya que demostró ser un método preciso que proporciona un equilibrio atractivo entre precisión y simplicidad conceptual (Shrive, Stuart, Quan \& Ghali, 2006). Además, se hallaron 8 casos atípicos que se eliminaron de los siguientes análisis. 
Los índices de asimetría y curtosis fueron excelentes (entre \pm 1.00 ) y la inspección gráfica de los datos no mostró alteraciones en la distribución dado que la mayor parte de los puntos del gráfico $Q-Q$ se encontraban sobre la diagonal. Finalmente, todas las variables y factores del cuestionario mostraron una significación mayor a .05 en la prueba de Levene, por lo que se asume que la variancia de las variables es homogénea en todos los niveles de la variable independiente.

ANÁLISIS DE LA ESTRUCTURA INTERNA DEL CSAI2R. ANÁlisis FACtORIAL CONFIRMATORIO

El ajuste a los datos fue satisfactorio (CFI .926, TLI .917, RMSEA .075, 90\% CI .069 .082, WRMR 1.423). Los pesos de regresión estandarizados $(p \leq .05)$ oscilaron entre .48 y .85 para el factor AS; entre .25 y .77 para el factor AC y entre .69 y .87 para el factor ACF. Debido a que los ítems 8, 15 y 18 presentaron un peso factorial por debajo del establecido (.40) se decidió reemplazar los ítems 8 y 15 y eliminar el 18 del análisis ya que este último no poseía reemplazo por ser un ítem generado ad-hoc en los procesos de entrevistas cognitivas.

De este modo, el ajuste mejoró levemente (CFI .954, TLI .948 RMSEA .062, 90\% CI .055 .070, WRMR 1.199) (ver Figura 1).

\section{CONSISTENCIA INTERNA}

El CSAI-2R mostró índices satisfactorios de confiabilidad compuesta. La consistencia interna de los factores osciló entre .90 (ACF) y .89 para AS y .84 para AC. A nivel general, el índice fue igual a .96 (ver Tabla 2).

\section{ESTUDIO DE DIFERENCIAS ENTRE GRUPOS}

Se hallaron diferencias estadísticamente significativas entre los sexos en todos los factores del inventario (ver Tabla 2). Además, en función de la modalidad deportiva (individual y / o grupal) también se hallaron diferencias estadísticamente significativas en los niveles de AS y AC, no así para ACF y los niveles de ANS total del inventario (ver Tabla 3). El tamaño del efecto para todos los análisis fue pequeño.

Por último, se analizó el puntaje total y por factores del CSAI-2R según el deporte practicado hallando un efecto significativo entre el deporte y los niveles de ansiedad pre-competitiva en los factores AS y AC. Ambos efectos son pequeños. La prueba post-hoc de Tukey indicó, en cuanto al factor AS que los/as deportistas de artes marciales poseen niveles significativamente mayores que los futbolistas y los jugadores de hockey. En relación al factor AC, los jugadores de hockey poseen niveles significativamente mayores de ansiedad que los deportistas de artes marciales (ver Tabla 4).

\section{Discusión}

Se adaptó el Inventario CSAI-2R a población deportiva argentina y se analizaron diferencias entre grupos en función de los antecedentes revisados con el objetivo de contribuir a la medida y estudio de los procesos afectivos en el contexto deportivo de Argentina.

Así, por una parte, mediante un proceso de AFC se halló que el CSAI-2R conservó su estructura factorial original, ratificando la estructura multidimensional de este proceso. Por último, los análisis de consistencia interna realizados arrojaron resultados de moderados a altos que oscilaron entre .84 y .96 . De los 21 reactivos finales, 5 fueron generados ad-hoc tomando como base los datos aportados por las entrevistas cognitivas. De estos 5 , 2 son ítems que reemplazan a los originales 8 y 15 del inventario. Adaptaciones previas también hallaron dificultades en el ítem 15 que fueron atribuidas a la traducción del término clammy (Lois Río \& Andrade Fernández, 2013). Esto mismo podría aplicarse al ítem 8, el cual presenta la dificultad idiomática en la frase Estoy preocupado/a de sentirme ahoga- 
do bajo presión que no sólo puede atribuirse a un síntoma de AC (sentirse preocupado) sino también a uno de AS (ahogado) que es una sensación corporal.

Esto demuestra la necesidad de adaptar las pruebas a cada contexto de aplicación en particular ya que los ítems funcionan distintos según el contexto, como así también la utilidad de procedimientos como por ejemplo, las entrevistas cognitivas en la adaptación de un instrumento a una cultura distinta de la cultura de origen de un test.

Por otra parte, en cuanto al estudio de diferencias entre grupos se halló que ciertas variables sociodemográficas están relacionadas con la ANS competitiva corroborando, en parte, que los componentes de la respuesta ansiosa son independientes entre sí en tanto tienen distintos antecedentes (Mellalieu et al., 2009). Así, estos resultados echan luz sobre la necesidad de incorporar variables sociodemográficas en el estudio de la ANS como indicó Parnabas (2015).

Primero, ambos sexos tienen puntuaciones medias más altas en ACF que en los demás factores, al igual que lo hallado por Botelho Vinhais y Salselas (2013). En este estudio las diferencias encontradas son estadísticamente significativas, siendo los varones quienes presentan niveles más altos de $\mathrm{ACF}$ tal como se hipotetizó y hallaron Pineda Espejel y colaboradores (2015). Asimismo, coincidiendo con los estudios de Kumar (2016) y Parnabas (2015), los varones mostraron niveles medios más bajos de ANS general que las mujeres, aunque no fue una diferencia significativa. Por último, una diferencia con otras investigaciones que analizaron la relación entre estas variables es que en el estudio que se informa las mujeres poseen niveles significativamente más altos en $\mathrm{AS}$ y más bajos de $\mathrm{AC}$ que los varones, confirmándose parcialmente la hipótesis planteada.

Una posible explicación de estas diferencias puede deberse a que las mujeres poseen mayor disposición a informar sus sentimientos en tanto gozan de mayor aceptación social (Lois Río \& Andrade Fernández, 2013; Pineda Espejel et al., 2015). Además, estas diferencias indican la necesidad de considerar esta variable para generar acciones de intervención y programas de entrenamiento en estrategias de afrontamiento apropiados a cada sexo que optimicen el rendimiento deportivo (Parnabas, 2015).

Segundo, en relación al deporte y modalidad deportiva se encontraron diferencias entre los grupos que van en línea con lo hallado por Lois Río y Andrade Fernández (2013) y Kirkby y Liu (1999) quienes mencionaron que los deportistas de artes marciales (deporte individual de contacto) mostraron mayores niveles de AS que los de modalidades colectivas (hockey y fútbol). Esto puede deberse a que los niveles de AS están vinculados con el grado de activación requerido por la tarea específica de cada deporte. Además, a diferencia de Botelho Vinhais y Salselas (2013) que encontraron que los deportes individuales tienen mayores niveles de AC aunque no son diferencias estadísticamente significativas, en el presente estudio los niveles de AC fueron significativamente más altos para las modalidades colectivas. Por último, no se hallaron diferencias en los niveles de ACF en ambas modalidades aunque fue el puntaje medio más alto tanto en deportes individuales como colectivos, a diferencia de lo reportado por Kirkby y Liu (1999) y Lois Río y Andrade Fernández (2013).

Una posible explicación de estas diferencias podría ser la distribución diferencial de la responsabilidad en los distintos deportes, ya que en los de equipo la responsabilidad del resultado se distribuye entre todos los miembros del equipo, no así en los individuales en los que la responsabilidad es de una sola persona y todas las evaluaciones recaen sobre ella. No obstante, aún en los deportes de equipo hay situaciones que dependen del rendimiento individual y, por tanto, pueden afectar los posibles efectos de la evaluación social a niveles similares a los que ocurren en los deportes individuales (Botelho Vinhais \& Salselas, 2013). Además, competir en un deporte colectivo podría hacer crecer la posibilidad de decepcionar a los compañeros si no se rinde al nivel esperado, lo cual también podría aumentar los niveles de ANS (Ramis et al., 2013). 
Estos resultados evidencian que el CSAI2R es una herramienta adecuada para evaluar ansiedad-estado competitiva. Martinent, Ferrand, Guillet y Gautheur (2009) indican que la adquisición de conocimiento se sustenta en la utilización de instrumentos de evaluación que permitan interpretaciones válidas y fiables en un ámbito concreto, por lo que construir o adaptar un inventario es un paso importante para estudiar el concepto de ansiedad estado competitiva en esta área del comportamiento.

Se espera que futuros estudios puedan superar algunas limitaciones que presentó este trabajo como el tipo de muestreo utilizado y / o poner en consideración variables como la edad de los deportistas o los niveles de experiencia de la muestra utilizada, ya que otros estudios las consideraron y mostraron niveles diferenciales de ANS competitiva (Rani \& Dhadwal, 2013). Además, se sugiere la posibilidad de incluir nuevas modalidades deportivas como deportes extremos o de riesgo, considerar el vínculo de la ansiedad-estado con otros constructos como podrían ser la autoeficacia o las lesiones deportivas. A su vez, para ofrecer evidencias externas de validez una medida a considerar podría ser la medida de rasgo de ansiedad o los estados de ánimo ya que se hallaron correlaciones positivas y moderadas entre factores de tensión y cólera evaluados mediante el POMS (Lois Río \& Andrade Fernández, 2013).

Por último, es importante destacar las implicancias que podría tener este estudio para la práctica profesional. Contar con una herramienta adaptada a población deportiva argentina para evaluar ANS estado competitiva posibilita conocer el comportamiento de esta variable en deportistas argentinos y diseñar estrategias de intervención acordes a la cultura y población evaluada.

Los deportistas podrían ser instruidos en relación a la ANS competitiva para posibilitar el desarrollo de evaluaciones reflexivas y racionales en relación con sus experiencias durante la competición y, de este modo, regularla. Para finalizar, la investigación en Psicología del Deporte en Argentina no es abundante, por lo que este tipo de aportes son significativos para el desarrollo del área y el conocimiento local específico (Pinto \& Vázquez, 2013). Así, se espera que futuras investigaciones tomen este trabajo como un punto de partida para mejorar y continuar desarrollando estudios en Psicología del Deporte en Argentina.

TABLA 1

DISTRIBUCIÓN DE LA MUESTRA SEGÚN SEXO Y DEPORTE

\begin{tabular}{|l|cccc|c|}
\hline \multirow{2}{*}{ Sexo } & \multicolumn{5}{|c|}{ ¿Qué deporte practicas? } \\
& Tenis & Fútbol & Hockey & Artes marciales & Total \\
\hline Femenino & 7 & 57 & 66 & 32 & 162 \\
Masculino & 7 & 66 & 62 & 60 & 195 \\
Total & 14 & 123 & 138 & 92 & 357 \\
\hline
\end{tabular}


TABLA 2

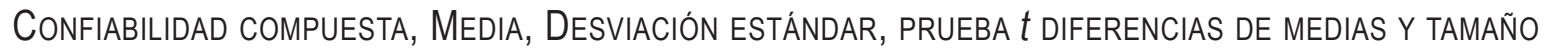
DEL EFECTO DE LAS MEDIDAS CSAI-2R SEGÚN SEXO

\begin{tabular}{|l|c|cc|cc|ccc|}
\hline \multirow{2}{*}{ Factores } & \multicolumn{2}{|c|}{ Femenino } & \multicolumn{2}{c|}{ Masculino } & \multicolumn{2}{c|}{} \\
& $p$ & $M$ & $D S$ & $M$ & $D S$ & $t$ & $\rho$ & $d$ \\
\hline Autoconfianza & .90 & 24.55 & 4.67 & 36.01 & 4.69 & -2.87 & .000 & -.31 \\
A. Somática & .89 & 10.86 & 3.47 & 9.61 & 2.65 & 3.81 & .001 & -.40 \\
A. Cognitiva & .84 & 11.32 & 3.73 & 10,12 & 3.16 & 3.24 & .004 & .34 \\
CSAI-2R & .96 & 46.73 & 6.34 & 45.73 & 5.81 & 1.53 & .126 & .16 \\
& & & & & & & &
\end{tabular}

TABLA 3

Media, DESVIACIÓn ESTÁNDAR, PRUEBA $t$ DIFERENCIAS DE MEDIAS Y TAMAÑo DEL EFECTO DE LAS MEDIDAS CSAI-2R SEGÚN LA MODALIDAD DEPORTIVA

\begin{tabular}{|l|cc|cc|ccc|}
\hline \multirow{2}{*}{ Factores } & \multicolumn{2}{|c|}{ Individual } & \multicolumn{3}{c|}{ Grupal } & \multicolumn{2}{c|}{} \\
& $M$ & $D S$ & $M$ & $D S$ & $t$ & $\rho$ & $d$ \\
\hline & & & & & & & \\
Autoconfianza & 25.12 & 4.82 & 25.45 & 4.71 & -.60 & .547 & -.06 \\
A. Somática & 10.89 & 3.39 & 9.87 & 2.94 & 2.83 & .005 & .32 \\
A. Cognitiva & 9.94 & 3.32 & 10.94 & 3.48 & -247 & .014 & -.29 \\
CSAI-2R & 45.95 & 6.17 & 46.26 & 6.03 & -.43 & .664 & -.05 \\
\hline
\end{tabular}

TABLA 4

AnÁlisis de Variancia, Media, Desviación estándar y tamaño del efecto del CSAI-2R y sus SUBESCALAS SEGÚN EL DEPORTE PRACTICADO

\begin{tabular}{|c|c|c|c|c|c|c|c|c|c|c|c|}
\hline \multirow[t]{2}{*}{ Factores } & \multicolumn{2}{|c|}{ Tenis } & \multicolumn{2}{|c|}{ Fútbol } & \multicolumn{2}{|c|}{ Hockey } & \multicolumn{2}{|c|}{ A. marciales } & \multirow[t]{2}{*}{$F$} & \multirow[t]{2}{*}{$p$} & \multirow[t]{2}{*}{$\eta 2$} \\
\hline & $M$ & $D S$ & $M$ & $D S$ & $M$ & $D S$ & $M$ & $D S$ & & & \\
\hline Autoconfianza & 22 & $3 . .94$ & 25.98 & 4.51 & 24.91 & 4.86 & 25.61 & 4.78 & 3.57 & .014 & .17 \\
\hline A. Somática & 9.14 & 2.31 & 9.86 & 3.04 & 9.90 & 2.85 & 11.17 & 3.46 & 4.39 & .005 & .01 \\
\hline A. Cognitiva & 9.64 & 2.02 & 10.56 & 3.35 & 11.37 & 3.62 & 9.99 & 3.48 & 3.31 & .020 & .02 \\
\hline CSAI-2R & 100.47 & 27.30 & 103.37 & 29.25 & 100.23 & 24.27 & 109.58 & 29.77 & 4.11 & .007 & .02 \\
\hline
\end{tabular}


FIGURA 1

ANÁLISIS FACTORIAL CONFIRMATORIO DE LA ESCALA CSAI-2R COMPUESTA POR 21 ÍTEMS

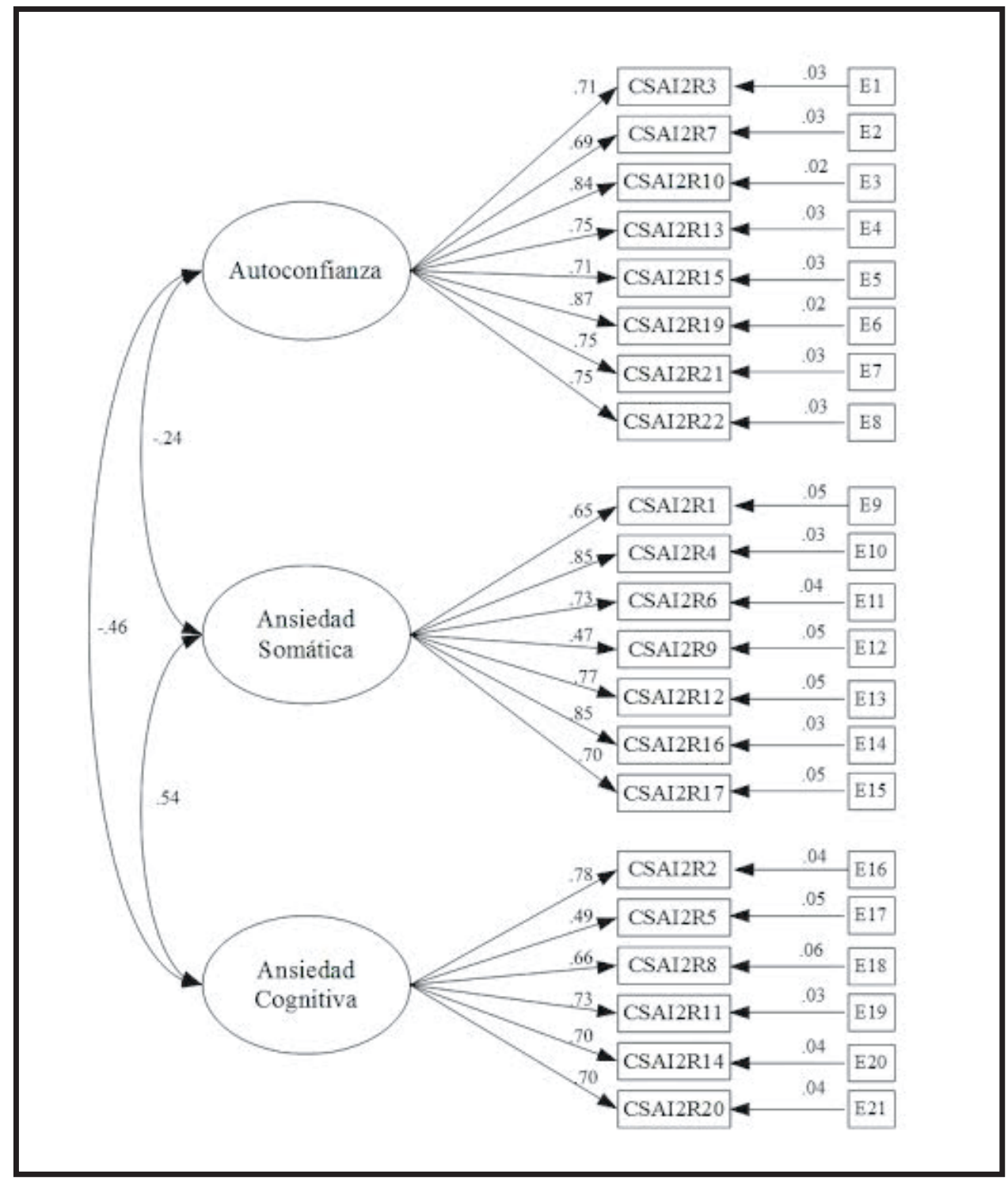

Todos los path fueron significativos $(p<.01)$ 


\section{REFERENCIAS BIBLIOGRÁFICAS}

American Educational Research Association (2014). Validity. En Standards for educational and psychological testing (pp. 11-31). Washington DC, United States of America: American Educational Research Association.

Andrade Fernández, E.M., Lois Río, G. \& Arce Fernández, C. (2007). Propiedades psicométricas de la versión española del Inventario de Ansiedad Competitiva CSAI-2R en deportistas [Psychometric properties of the Spanish version of Competitive State Anxiety Inventory-2R in Athletes]. Psicothema, 19(1), 150-155.

Arruza Gabilondo, J.A., González Rodríguez, O., Palacios Moreno, M., Arribas Galarraga, S. \& Cecchini Estrada, J.A. (2010). Validación del Competitive State Anxiety Inventory 2 Reducido (CSAI-2 RE) mediante una aplicación web [Web and reduce version of the Competitive State Anxiety Inventory-2 validation]. Revista Internacional de Medicina y Ciencias de la Actividad Física y el Deporte, 12(47), 539-556.

Azevedo-Cruz, J.F. (1996). Stress, ansiedade e rendimento na competiçao desportiva [Stress, anxiety and performance in sport competition]. Braga: Centro de Estudios em Educaçao e Psicología, Universidade do Minho.

Bologna. E. (2010). Estadística para psicología y educación [Statistics for psychology and education]. Córdoba: Brujas.

Botelho Vinhais, J. \& Salselas, V. (2013). Ansiedade pré-competitiva nas modalidades coletivas e individuais [Precompetitive anxiety in team sports and individual]. Tesis de Maestría no publicada. Universidade de Trás-os-Montes e Alto Douro, Portugal. Recuperado el 30 de agosto de 2016 https://repositorio.utad.pt/bitstream/10 348/4891/1/msc_jmb vinhais.pdf

Cohen, J. (1988). Statistical power analysis for the behavioral sciences (2a. ed.). Hillsdale, NY: Lawrence Earlbaum Associates.

Cox, R.H., Martens, M.P. \& Russell, W.D. (2003). Measuring anxiety in athletics: The Revised
Competitve State Anxiety Inventory-2. Journal of Sport \& Exercise Psychology, 25, 519-533.

Ekkekakis, P. (2012). Affect, mood and emotion. En G. Tenenbaum \& R. Eklund (Eds.), Measurement in Sport and Exercise Psychology (pp. 320-519). United States: Human Kinetics.

Gaetano, R., Gómez, P. \& Altavilla, G. (2015). Anxiety in the youth physical and sport activity. Mediterranean Journal of Social Sciences, 6(3), 227-230. http://doi.org/10.5901/mjss.2015.v6n $3 \mathrm{~s} 2 \mathrm{p} 227$

Graham, J.W. (2009). Missing data analysis: Making it work in the real world. Annual Review of Psychology, 60, 549-576.

George, D. \& Mallery, P. (2011). IBM SPSS Statistics 21 step by step: A simple guide and reference. Boston: Pearson Education.

Hammersmeister, J. \& Burton, D. (2001). Stress, appraisal, and coping revisited: Examining the antecedents of competitive state anxiety with endurance athletes. The Sport Psychologist, 15, 66-90.

Jones, G. (1995). More than just a game: Research developments and issues in competitive anxiety in sport. British Journal of Psychology, 86, 449478.

Kirkby, R.J. \& Liu, J. (1999). Precompetition anxiety in Chinese Athletes. Perceptual and Motor Skills, 88, 297-303.

Kumar, A. (2016). A study of pre-competitive anxiety involving male and female players competing in team versus individual events. International Journal of Physical Education, Sports and Health, 3(1), 135-137.

Larson-Hall, J. (2010). Describing data numerically and graphically and assessing assumptions for parametric tests. A guide to doing statistics in second language research using SPSS. New York: Routledge.

Lois Río, G. \& Andrade Fernández, E.M. (2013). Medida de la Ansiedad Precompetitiva: Adaptación del CSAI-2R al español [Pre-competitive Anxiety measure: Spanish adaptation of the CSAI-2R]. Tesis de Doctorado no publica- 
da. Universidad Santiago de Compostela, España. Recuperado el 4 de marzo de 2016 de http://www.tdx.cat/handle/10803/145583

Martens, R. (1974). Arousal and motor performance. En J. Wilmore (Ed.), Exercise and sport science review. New York: Academic Press.

Martens, R. (1977). Sport Competition Anxiety Test. Champaign, IL: Human Kinetics.

Martens, R., Burton, D., Rivkin, F. \& Simon, J. (1980). Reliability and validity of the Competitive State Anxiety Inventory (CSAI). En C.H. Nadeau, W.C. Halliwell, K.M. Newell \& G.C. Roberts (Eds.), Psychology of motor behavior and sport-1979 (pp. 91-99). Champaign, IL: Human Kinetics.

Martens, R., Vealey, R.S. \& Burton, D. (1990). Competitive anxiety in sport. Champaign, IL: Human Kinetics.

Martinent, G., Ferrand, C., Guillet, E. \& Gautheur, S. (2009). Validation of the French version of the Competitive State Anxiety Inventory-2 Revised (CSAI-2R) including frequency and direction scales. Psychology of Sport and Exercise, 11, 51-57. http://doi.org/10.1016/j.psych sport.2009.05.001

Mc Hugh, M.L. (2012). Interrater reliability: The kappa statistics. Biochemia Medica, 22(3), 276278.

Mellalieu, S.D., Hanton, S. \& Fletcher, D. (2009). A competitive anxiety review: Recent directions in sport psychology research. New York: Nova Science Publishers.

Meyers, L.S., Gamst, G.C. \& Guarino, A.J. (2013). Assessing distribution shape: Normality, skewness and kurtosis. Evaluating score distribution assumptions. En Performing Data Analysis Using IBM SPSS. United States: Wiley.

Mimura, C. \& Griffiths, P. (2008). A Japanese version of the Perceived Stress Scale: Crosscultural translation and equivalence assessment. BMC Psychiatry, 8(1), 85. http://doi.org/10.1 186/1471-244X-8-85.

Montero, I. \& León, O. (2007). A guide for naming research studies in Psychology. International
Journal of Clinical and Health Psychology, 7(3), 847-862.

Muñiz, J. \& Hambleton, R.K. (1996). Directrices para la traducción y adaptación de los test. $P a$ peles del Psicólogo, 66. Recuperado el 17 de marzo de 2013 de www.papelesdelpsicologo. es/imprimir.asp?id $=737$.

Nunnally, J.C. (1978). Psycometric theory. Nueva York: McGraw-Hill.

Parnabas, V. (2015). Identifying the gender differences on the level of competitive state anxiety and sport performance among track and field athletes. The International Journal of Indian Psychology, 2, 23-29.

Pineda Espejel, A., López-Walle, J. \& Tomas, I. (2015). Factores situacionales y disposicionales como predictores de la ansiedad y autoconfianza precompetitiva en deportistas universitarios [Situational and dispositional factores as pre-competitive anxiety and selfconfidence predictors in university athletes]. Cuadernos de Psicología del Deporte, 15(2), 55-70.

Pinto, M.F. \& Vázquez, N. (2013). Ansiedad estado competitiva y estrategias de afrontamiento: $\mathrm{Su}$ relación con el rendimiento en una muestra argentina de jugadores amateurs de golf [Competitive state anxiety and coping: Their relationship with performance in an amateur Argentinian golf sample]. Revista de Psicología del Deporte, 22(1), 47-52.

Ramis, Y., Torregrosa, M. \& Cruz, J. (2013). Revisitando a Simon \& Martens: La ansiedad competitiva en deportes de iniciación [Simon \& Martens revisited: Competitive anxiety in youth sports]. Revista de Psicología del Deporte, 22 (1), 77-83.

Rani, S. \& Dhadwal, M.K. (2013). Comparison of pre-competitive and post competitive anxiety level of inter-collegiate football players. International Journal of Movement Education and Social Sciences, 2(1), 1-4.

Shrive, F.M., Stuart, H., Quan, H. \& Ghali, W.A. (2006). Dealing with missing data in a multi- 
question depression scale: A comparison of imputation methods. BMC Medical Research Methodology, 6(1), 57. http://doi.org/10.1186/ 1471-2288-6-57.

Spielberger, C. D., Gorsuch, R. L. \& Lushene, R. E. (1970). The State-Trait Anxiety Inventory (Test Manual). Palo Alto, California: Consulting Psychologists Press.

Tabachnick. B.G. \& Fidell, L.S. (2011). Using Multivariate Statistics. Needham Heights: Pearson.

Vaiman, M. (2011). Entrevistas cognitivas [Cognitive interviews]. En M. Cupani, M. Vaiman,
M.F. Zalazar Jaime \& E.C. Marchesi (Eds.), Guía de trabajos prácticos para alumnos promocionales. Cátedra de Técnicas Psicométricas (pp. 24-27). Facultad de Psicología, Universidad Nacional de Córdoba: Argentina.

Yu, C.Y. \& Muthen, B. (2002, Abril). Evaluation of model fit indices for latent variable models with categorical and continuous outcomes. Trabajo presentado en el Annual Meeting of the American Educational Research Association, New Orleans, LA.

Centro de Investigaciones de la Facultad de Psicología (CIPsi) Grupo vinculado al Centro de Investigaciones y Estudios sobre Cultura y Sociedad (CIECS)

Consejo Nacional de Investigaciones

Cientificas y Técnicas (CONICET)

Universidad Nacional de Córdoba (UNC)

Córdoba - Républica Argentina

Facultad de Educación

Universidad Autónoma de Madrid (UAM)

Madrid - España

Fecha de recepción: 14 de junio de 2016

Fecha de aceptación: 25 de octubre de 2016 